\title{
Echocardiographic Changes in Chronic Kidney Disease Patients on Maintenance Hemodialysis
}

Farah Anum Jameel ${ }^{1}$, Abdul Mannan Junejo ${ }^{1}$, Qurat ul ain Khan ${ }^{1}$, Sudeep Date ${ }^{2}$, Ahmad Faraz ${ }^{3}$, Syed Hasan Mustafa Rizvi ${ }^{4}$, Fatima Ahmad ${ }^{5}$, Muhammad Tahir 6

1. Nephrology, Jinnah Postgraduate Medical Center, Karachi, PAK 2. Orthopaedics and Trauma, Cumberland Infirmary, Carlisle, GBR 3. Trauma and Orthopaedics, Leeds Teaching Hospitals NHS Trust, Leeds, GBR 4. Internal Medicine, Peterborough City Hospital, Peterborough, GBR 5. Anaesthesia, Punjab Institute of Cardiology, Lahore, PAK 6. Orthopaedics, Jinnah Postgraduate Medical Center, Karachi, PAK

Corresponding author: Muhammad Tahir, doctor.muhammad.tahir@gmail.com

\section{Abstract}

\section{Introduction}

Chronic kidney disease (CKD) carries a significant association with cardiac diseases, which suggests a minor reduction in the glomerular filtration rate (GFR) can act as an independent risk factor for causing cardiovascular abnormalities. Patients of CKD having cardiovascular disease (CVD) had three to thirty times higher risk of mortality as compared to the general population. In addition, mortality among cardiovascular patients has been found to be twofold higher in CKD stage 2 patients and three-fold higher in patients with stage $3 \mathrm{CKD}$, when collated to patients with normal renal function. Furthermore, cardiomyopathy among hemodialysis (HD) is due to the presence of coronary artery obstruction, reduction in coronary reserves, and left ventricular (LV) physiological-structural abnormalities secondary volume and pressure overload. Echocardiography is a gold standard diagnostic modality for the identification of cardiac structural and functional abnormalities. Therefore, the evaluation of echocardiographic parameters in patients of CKD can help to determine the risk and prognosis of CVD in patients of CKD. In the present study, we evaluated the echocardiographic findings in patients of CKD on maintenance hemodialysis.

\section{Methods}

This cross-sectional study was conducted in the nephrology unit of Jinnah Postgraduate Medical Center between March 2019 to October 2019. A total of 100 patients who were on maintenance for more than one year were included in the analysis. Two-dimensional transthoracic echocardiography was done in each patient for the determination of cardiac structural and functional parameters such as LV hypertrophy, LV systolic dysfunction, and LV diastolic dysfunction.

\section{Results}

The mean age of the patients was $46.9 \pm 12.8$ years. There was male dominance with male/female ratio $63 / 37$.

Received 06/25/2020

Review began 06/27/2020 Review ended 06/27/2020 Published 07/02/2020

(c) Copyright 2020

Jameel et al. This is an open access article distributed under the terms of the Creative Commons Attribution License CC-BY 4.0., which permits unrestricted use, distribution, and reproduction in any medium, provided the original author and source are credited. There were $39 \%$ hypertensive and $62 \%$ anemic patients. LV dysfunction was diagnosed in $31 \%$ of patients, LV diastolic dysfunction in $47 \%$ patients, and left ventricular hypertrophy (LVH) in $55 \%$ of patients. $\mathrm{LVH}$ was found in $74.3 \%$ hypertensive patients versus only $42.6 \%$ non-hypertensive patients (p-value 0.001 ). LV systolic dysfunction was also high in hypertensive patients, $46.1 \%$ versus $21.3 \%$ patients in nonhypertensive patients (p-value 0.008).

\section{Conclusion}

There is a high frequency of cardiac functional and structural abnormalities in CKD patients on HD especially in patients having concomitant hypertension. LVH is the most common structural defect and LV diastolic dysfunction is the most common functional cardiac defect in CKD patients on hemodialysis.

Categories: Cardiology, Internal Medicine, Nephrology

Keywords: chronic kidney disease, maintenance hemodialysis, echocardiography

\section{Introduction}

Chronic kidney disease (CKD) carries a significant association with cardiac diseases, which suggests a minor reduction in the glomerular filtration rate (GFR) is an independent risk factor for causing cardiovascular abnormalities [1]. Manjunath et al. reported that patients with GFR $\leqslant 59 \mathrm{~mL} / \mathrm{min} / 1.73 \mathrm{~m} 2$ have a $38 \%$ higher risk of cardiovascular disease (CVD) development as compared to those having GFR $\geqslant 90 \mathrm{~mL} / \mathrm{min} / 1.73 \mathrm{~m} 2$ [1]. CVD is associated with $>50 \%$ of the deaths in CKD patients. Patients of CKD having CVD had three to thirty times higher risk of mortality as compared to the general population [2].In addition, mortality among cardiovascular patients has been found to be twofold higher in CKD stage 2 patients and three-fold higher in patients with stage $3 \mathrm{CKD}$, when collated to patients with normal renal function [3]. 
Left ventricular hypertrophy (LVH) is one of the common structural cardiac defects in CKD patients. LVH significantly increases the risk of cardiac ischemia, heart failure, and is a strong predictor of mortality in CKD patients [4]. LV dysfunction is an initial precursor of CVD and leads to LVH in the follow-up period [5]. Furthermore, cardiomyopathy among hemodialysis (HD) is due to the presence of coronary artery obstruction, reduction in coronary reserves, and left ventricular physiological-structural abnormalities secondary volume and pressure overload [6]. When efforts to reduced left cardiac preload are not made, adaptation in left ventricular is activated, which leads to a decrease in capillary density, diastolic dysfunction, and disturbances in intraventricular conduction, dilatation, and more compensatory hypertrophy [7].

These phenomena increase vulnerability to increase electrical excitability, leading to sudden cardiac death among these patients [8]. Echocardiography is a gold standard diagnostic modality for the determination of cardiac structural and functional abnormalities. Therefore, the evaluation of echocardiographic parameters in patients of CKD can help to determine the risk and prognosis of CVD in patients of CKD [9]. In the present study, we evaluated the echocardiographic findings in patients of CKD on maintenance HD.

\section{Materials And Methods}

This cross-sectional study was conducted in the nephrology unit of Jinnah Postgraduate Medical Center within 07 months duration from March 2019 to October 2019. A total of 100 patients who were on maintenance for more than one year were included in the analysis. Patients who were already known for having CVD such as coronary artery disease, valvular heart disease congenital heart, or primary cardiomyopathy were excluded. Approvalafromahospital ethical committee was taken. Written consent for participation of the study was also signed by each patient.

In all patient's baseline laboratory investigations such as complete blood count, renal parameters, lipid profile were measured in each patient as a routine investigation protocol. Two-dimensional echocardiography was done in each patient for the determination of cardiac structural and functional parameters. Patients having LV ejection fraction $(\mathrm{EF})<50 \%$ were diagnosed with having $\mathrm{LV}$ dysfunction. For determination of $\mathrm{LV}$ diastolic dysfunction, the $\mathrm{E} / \mathrm{A}$ ratio was calculated using Doppler velocity measurements, E/A ratio $<0.75$, or $>1.8$ was labeled as LV diastolic dysfunction. Patients having an intraventricular thickness or LVaposterior wall thickness $\geqslant 12 \mathrm{~mm}$ were diagnosed as having $\mathrm{LV}$ hypertrophy(LVH).

Already known cases of hypertension or patients having BP $\geqslant 140 / 90$ were labeled as hypertensive. While anemia was diagnosed if the hemoglobin level was $<135 \mathrm{gm} / \mathrm{dL}$ in male patients and $<12.5 \mathrm{gm} / \mathrm{dL}$ in female patients.

Data were analyzed in SPSS version 23.0 (IBM, Armonk, USA). The Pearson Chi-square test was applied to determine the association of anemia and hypertension with cardiac functional and structural abnormalities. $\mathrm{p}$-Value $\leqslant 0.05$ was taken as significant.

\section{Results}

The mean age of patients was $46.9 \pm 12.8$ years. There was male dominance with male/female ratio 63/37. There were $39 \%$ hypertensive and $62 \%$ anemic patients (Table 1 ).

\begin{tabular}{|l|l|}
\hline Variable & Value \\
\hline Mean Age & $46.9 \pm 12.8$ (Range; 21-82) \\
Gender (Male/Female) & $63 / 37$ \\
Serum Creatinine (mg/dL) & $5.36 \pm 1.49$ \\
Diabetes Mellitus & $32 \%$ \\
Hypertension & $39 \%$ \\
Anemia & $62 \%$ \\
\hline
\end{tabular}

TABLE 1: Baseline Characteristics of Study Participants

Regarding echocardiographic abnormalities, LV dysfunction was diagnosed in 31\% patients, LV diastolic dysfunction in $47 \%$ patients and LVH in $55 \%$ of the patients (Figure 1 ). 


\section{Cureus}

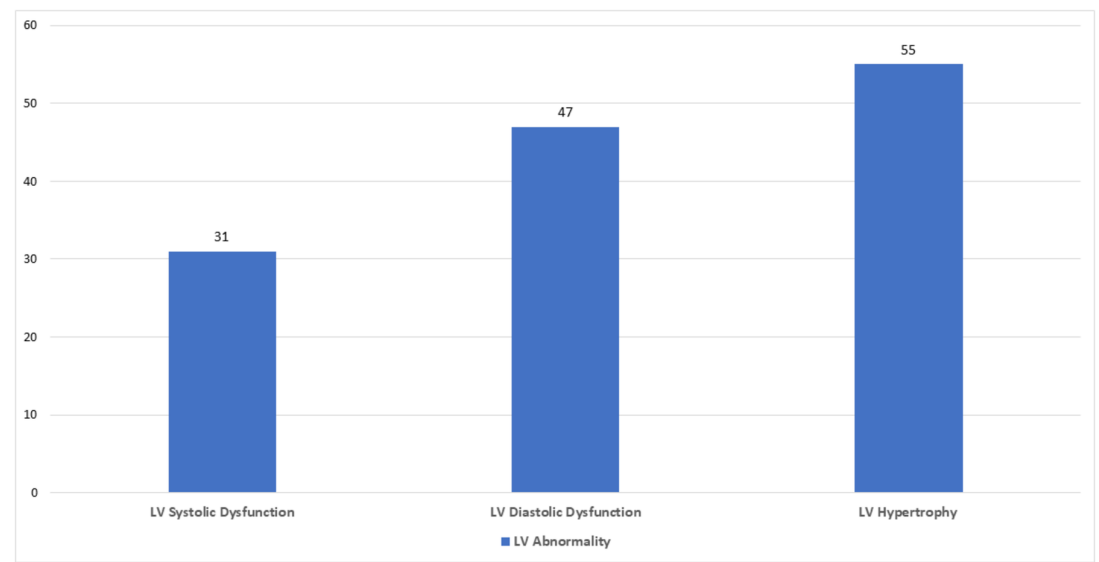

FIGURE 1: Echocardiographic Abnormalities in patients of CKD

CKD- Chronic Kidney Disease, LV- Left Ventricle

There was higher frequency of LV systolic dysfunction and LVH in patients having hypertension as compared to non-hypertensive patients (p-value $0.008 \& 0.001$ respectively) (Table 2).

\begin{tabular}{|c|c|c|c|}
\hline & Hypertension (N=39) & Non-Hypertension $(\mathrm{N}=61)$ & p-Value \\
\hline LV Systolic Dysfunction & $18(46.1 \%)$ & $13(21.3 \%)$ & 0.008 \\
\hline LV Diastolic Dysfunction & $22(56.4 \%)$ & $25(40.9 \%)$ & 0.13 \\
\hline LVH & $29(74.3 \%)$ & $26(42.6 \%)$ & 0.001 \\
\hline
\end{tabular}

\section{TABLE 2: Comparison of Echocardiographic Abnormalities in Hypertensive and Non-Hypertensive}

\section{Patients}

LV- left ventricular, LVH- left ventricular hypertrophy

\section{Discussion}

CKD patients have higher proportions of congestive heart failure that is associated with a higher mortality rate in these patients [5]. Echocardiography is a valuable tool to assess the assess changes in function and structure of the heart that result from CKD. Abnormal LV geometry, reduction in interventricular septum strength, and changes in LV mass index are important parameters that are affected by CKD in patients with preserved EF [6].

Previous studies have reported anemia, volume overload, electrolyte abnormalities edema, and hypertension as risk factors that alter the risk of CVD in CKD patients [7,8]. In the present study, anemia was diagnosed in $62.0 \%$ of patients, hypertension in $39 \%$, and diabetes mellitus in $32 \%$ of patients. A study by Tsilonis et al. reported diabetes mellitus in $24 \%$ of patients and hypertension in $22 \%$ of patients of CKD patients on HD [9].

The current study reports the most common cardiac abnormality was $\mathrm{LVH}$, found in $55 \%$ of patients, followed by LV diastolic dysfunction in $47 \%$ patients and LV systolic dysfunction in $31 \%$ of patients. A study conducted by Shivendra et al. reported LVH in $48 \%$ of patients, diastolic dysfunction in $51.42 \%$ patients, and systolic dysfunction in $28.57 \%$ patients of CKD on maintenance HD [10]. Agarwal et al. reported LV diastolic dysfunction in $53.2 \%$ patients and LV systolic dysfunction in $30 \%$ of patients having severe CKD [4]. Another study by Laddha et al. reported LVH in 74.3\% patients, LV diastolic dysfunction in $61.4 \%$ patients, and systolic dysfunction in $24.3 \%$ patients [11]. A similar study by Ahmed et al. LVH in $80 \%$ of patients, LV diastolic dysfunction in $53.3 \%$ patients, and LV systolic dysfunction in $36.3 \%$ patients [12].

Some studies have reported LV systolic dysfunction in all patients of HD $[13,14]$ which is very high as compared to our study and the above-mentioned studies. The possible reason for this high proportion may 
be that these studies used the positron emission tomography scan for determination of systolic dysfunction that uses contrast-induced ischemic changes for diagnosis of ischemia and is superior to echocardiography for determination of cardiac dysfunction [14].

In our study, hypertension was found a significant risk factor for cardiac abnormalities. In our study, LVH was found in $74.3 \%$ hypertensive patients versus in only $42.6 \%$ non-hypertensive patients. Similarly, LV systolic dysfunction was also high in hypertensive patients, $46.1 \%$ versus $21.3 \%$ patients in nonhypertensive patients. Barre et al. also reported similar findings; they reported LVH in $64.47 \%$ hypertensive patients and in 33.3\% non-hypertensive patients (20) [3]. Shivendra et al. also reported similar findings; they reported LVH in 51\% hypertensive versus in only $8.57 \%$ normotensive patients (21) [10].

In summary, cardiac abnormalities are common in patients of CKD on maintenance HD. So echocardiography should be performed at regular intervals in these patients especially those having hypertension along with CKD. A timely diagnosis of cardiac abnormalities can help in the early management of these complications and can help to reduce cardiac induced morbidity and mortality in CKD patients.

\section{Conclusions}

In conclusion, structural and physiological abnormalities of right and left ventricle are more commonly affected among patients with mild or moderate renal disorder, whereas right ventricle function and structure are independently associated with CKD progression. Furthermore, there is higher frequency of cardiac abnormalities among CKD patients on maintenance HD especially in patients having concomitant hypertension. LVH is the most common structural defect and LV diastolic dysfunction is the most common functional cardiac defect in CKD patients on HD. Additional workup of cardiovascular pharmacological management specifically among the HD group is required to make an evidence-based clinical decision to alleviate CVD in this higher risk group. Technology advances in HD may provide an opportunity to prevent CVD associated with dialysis treatment.

\section{Additional Information \\ Disclosures}

Human subjects: Consent was obtained by all participants in this study. Medical Ethics Review Board Committee issued approval 19350/2019. With reference to your application/letter dated 29th May, 2019, on the subject "Echocardiographic Changes in Chronic Kidney Disease Patients on Maintenance Hemodialysis" and to say that the Institutional Review Board has approved your subject proposal and allowed to retrieve data for publication. Animal subjects: All authors have confirmed that this study did not involve animal subjects or tissue. Conflicts of interest: In compliance with the ICMJE uniform disclosure form, all authors declare the following: Payment/services info: All authors have declared that no financial support was received from any organization for the submitted work. Financial relationships: All authors have declared that they have no financial relationships at present or within the previous three years with any organizations that might have an interest in the submitted work. Other relationships: All authors have declared that there are no other relationships or activities that could appear to have influenced the submitted work.

\section{References}

1. Manjunath G, Tighiouart H, Ibrahim H, et al.: Level of kidney function as a risk factor for atherosclerotic cardiovascular outcomes in the community. J Am Coll Cardiol. 2003, 41:47-55. 10.1016/S07351097(02)02663-3

2. Muntner P, Judd SE, Gao L, et al.: Cardiovascular risk factors in CKD associate with both ESRD and mortality. J Am Soc Nephrol. 2013, 24:1159-1165. 10.1681/ASN.2012070642

3. Parfrey PS, Foley RN, Harnett JD, Kent GM, Murray DC, Barre PE: Outcome and risk factors for left ventricular disorders in chronic uraemia. Nephrol Dial Transplant. 1996, 11:1277-1285. 10.1093/ndt/11.7.1277

4. Agarwal S, Dangri P, Kalra OP, Rajpal S: Echocardiographic assessment of cardiac dysfunction in patients of chronic renal failure. J Indian Acad Clin Med. 2003, 4:296-303.

5. McAlister FA, Ezekowitz J, Tonelli M, Armstrong PW: Renal insufficiency and heart failure: prognostic and therapeutic implications from a prospective cohort study. Circulation. 2004, 109:1004-1009. 10.1161/01.CIR.0000116764.53225.A9

6. Gori M, Senni M, Gupta DK, et al.: Association between renal function and cardiovascular structure and function in heart failure with preserved ejection fraction. Eur Heart J. 2014, 35:3442-3451. 10.1093/eurheartj/ehu254

7. El Arbagy AR, Koura MA, El Barbary HS, Abou El Nasr AE: Comparative study of the effect of high-flux versus low-flux dialysis membranes on metabolic abnormalities in chronic hemodialysis patients. Menoufia Med J. 2014, 27:677-682. 10.4103/1110-2098.149667

8. Hayashi SY, Rohani M, Lindholm B, et al.: Left ventricular function in patients with chronic kidney disease evaluated by colour tissue Doppler velocity imaging. Nephrol Dial Transplant. 2005, 21:125-132. 10.1093/ndt/gfi075

9. Tsilonis K, Sarafidis PA, Kamperidis V, et al.: Echocardiographic parameters during long and short interdialytic intervals in hemodialysis patients. Am J Kidney Dis. 2016, 68:772-781. 


\section{Cureus}

10.1053/j.ajkd.2016.06.017

10. Shivendra S, Doley PK, Pragya P, Sivasankar M, Singh VP, Neelam S: Echocardiographic changes in patients with ESRD on maintenance hemodialysis-a single centre study. J Cardiovasc Dis Diagn. 2014, 2:4.

10.4172/2329-9517.1000165

11. Laddha M, Sachdeva V, Diggikar PM, Satpathy PK, Kakrani AL: Echocardiographic assessment of cardiac dysfunction in patients of end stage renal disease on haemodialysis. J Assoc Physicians India. 2014, 62:2832.

12. Ahmed HA, Yassein YS, Zaki SA, Al Qersh AM, Fahim FS: Study of echocardiographic changes among adult patients on maintenance hemodialysis. Menoufia Med J. 2016, 29:44-51. 10.4103/1110-2098.178949

13. McIntyre CW, Burton JO, Selby NM: Hemodialysis-induced cardiac dysfunction is associated with an acute reduction in global and segmental myocardial blood flow. Clin J Am Soc Nephrol. 2008, 3:19-26. 10.2215/CJN.03170707

14. Siqueira TMA, Ferreira PAM, Monteiro FDC Jr, et al.: Echocardiographic parameters as cardiovascular event predictors in hemodialysis patients [Article in Spanish]. Arq Bras Cardiol. 2012, 99:714-723. 10.1590/s0066$782 \times 2012005000065$ 